\title{
Two-dimensionality of gravity water flows governed by the equatorial $f$-plane approximation
}

\author{
Calin Iulian Martin ${ }^{1}$
}

Received: 24 February 2017 / Accepted: 17 April 2017 / Published online: 22 April 2017

(C) The Author(s) 2017. This article is an open access publication

\begin{abstract}
We show that gravity wave trains governed by the equatorial $f$-plane approximation propagate at the free surface of a rotational water flow of constant vorticity vector $\left(\Omega_{1}, \Omega_{2}, \Omega_{3}\right)$ over a flat bed only if the flow is two-dimensional. Owing to the presence of Coriolis effects, our result is also true even if the vorticity vector vanishes. This represents a striking difference when compared with the cases without geophysical effects discussed in Constantin (Europhys Lett 86:29001, 2009, Eur J Mech 30:12-16; 2011) and Martin (J Math Fluid Mech 2016. doi:10.1007/s00021-016-0306-1), where the conclusion about the two-dimensionality of the flow was possible under the assumption of constant nonvanishing vorticity vector. Another upshot is that the only nonzero component of the vorticity that may not vanish is $\Omega_{2}$, that is, the one pointing in the horizontal direction orthogonal to the direction of wave propagation.
\end{abstract}

Keywords $f$-plane approximation · Gravity wave trains · Vorticity

Mathematics Subject Classification Primary 35Q31 · 35Q35; Secondary 35Q86

\section{Introduction}

There is an increased interest nowadays in the study of geophysical water flows. These are flows whose motion is influenced by the Earth's rotation reflected through the manifestation of the Coriolis force. The thorough understanding of geophysical flows is highly dependent upon inclusion of nonlinear aspects that considerably complicate the matter to the point of eluding exact treatment; this drawback is somehow mitigated by the discovery by Gerstner [17] who presented in 1802 the only known explicit exact solution (in Lagrangian variables) for the governing equations for periodic homogeneous two-dimensional traveling gravity

Calin Iulian Martin

calin.martin@univie.ac.at

1 Faculty of Mathematics, University of Vienna, Oskar-Morgenstern-Platz 1, 1090 Vienna, Austria 
water waves. Gerstner solution was extended in 1932 to a heterogeneous fluid by DubreilJacotin [13]; we refer the reader to Constantin [1] and Henry [18] for modern presentations of the Gerstner wave solution.

The specific general difficulties raised by the nonlinear character of equations governing the water wave propagation are enhanced by the inclusion of the before mentioned Coriolis effects; especially the complex dynamics of flows near the equatorial Pacific presents some peculiarities like a strong stratification (evidentiated by the presence of an interface, called thermocline, separating two layers of constant density), the presence strong depth-dependent underlying currents, cf. [9], with flow reversal at a depth of about 100-200 m and a wide range of other wave propagation phenomena. The softening of these intricacies becomes possible through the employment of suitable approximations of the geophysical governing equations; one of these is the $f$-plane approximation which is appropriate for oceanic flows within a restricted meridional range of about $2^{\circ}$ latitude from the Equator [9, 12,21].

The rigorous mathematical study of geophysical water flows has resulted in the appearance of a bulk of papers commencing with the modeling of wave-current interactions in the $f$ plane approximation for underlying currents of constant vorticity, by Constantin [4] and with the derivation of an exact solution (in the Lagrangian framework) of the nonlinear geophysical water waves in the $\beta$-plane near the Equator by Constantin in [5] by a suitable and substantial modification of the Gerstner wave solution to the case of three-dimensional flows. These studies were followed by papers presenting explicit exact solutions describing flows accommodating flow reversal and allowing for underlying currents in the $\beta$-plane setting, cf. [7,8,19]. An additional aspect of centrifugal forces in the $\beta$-plane approximation was addressed recently by Henry in [22] where an exact and explicit solution describing equatorially trapped waves was obtained.

Circumscribed to the same vein of solution flows exhibiting a prefered direction of propagation are recent works describing oceanic flows propagating in an azimuthal direction and exhibiting a complicated vertical structure $[10,11,25]$. Such geophysical flows were also analyzed from the perspective of stability/instability properties [6,16,20,23,24].

Our intention here is to show that gravity wave trains can propagate at the free surface of a rotational water flow of constant vorticity and governed by the equatorial $f$-plane approximation only if the flow has a two-dimensional character. From a historical perspective, the property that the flow below a wave train does not vary in the horizontal direction perpendicular to the direction of motion was validated by previous works of Constantin and Kartashova [2] proving the two-dimensionality of constant vorticity water flows below wave trains driven by capillarity and of Constantin [3] showing the two-dimensionality of constant vorticity gravity flows below wave trains; see also the works of Wahlén [30] and Stuhlmeier [29], the latter in the case of solitary waves.

\section{The governing equations}

We choose a rotating framework with the origin at a point on the Earth's surface, with the $x$ axis pointing horizontally due East, the $y$-axis horizontally due North, while the $z$-axis is oriented upward. We will consider here regular wave trains of water waves propagating steadily in the direction of the horizontal $x$ axis, periodic (of period L) in the variable $x$, and exhibiting no variation in the $y$ direction. We will, in fact, show that the lack of the $y$-dependence of the free surface manifests throughout the entire flow. 
The fluid domain is bounded below by the impermeable flat bed $z=-d$ and above by the free surface $z=\eta(x-c t)$, where the function $\eta$ gives the wave profile and $c>0$ is the wave speed.

If $(u, v, w)$ denotes the velocity field, $t$ is the time variable, $P$ the pressure, $\rho$ the constant density, $g$ is the constant acceleration of gravity, and $\omega=73 \times 10^{-6} \mathrm{rad} / \mathrm{s}$ is the constant rotational speed of Earth round the polar axis, the governing equations in the $f$-plane approximation near the Equator are the Euler's equations

$$
\begin{aligned}
u_{t}+u u_{x}+v u_{y}+w u_{z}+2 \omega w & =-\frac{1}{\rho} P_{x} \\
v_{t}+u v_{x}+v v_{y}+w v_{z} & =-\frac{1}{\rho} P_{y} \\
w_{t}+u w_{x}+v w_{y}+w w_{z}-2 \omega u & =-\frac{1}{\rho} P_{z}-g
\end{aligned}
$$

and the equation of mass conservation

$$
u_{x}+v_{y}+w_{z}=0 .
$$

To single out the water wave problem from a vast range of hydrodynamical ones we impose the kinematic boundary conditions

$$
w=(u-c) \eta_{x} \text { on } \quad z=\eta(x-c t),
$$

and

$$
w=0 \text { on } z=-d,
$$

together with the dynamic boundary condition

$$
P=P_{\text {atm }} \quad \text { on } \quad z=\eta(x-c t) .
$$

To model observed phenomena, like underlying currents, it is essential to include the vorticity $\Omega(x, y, z, t)$, obtained as the curl of the velocity field, that is,

$$
\Omega=\left(w_{y}-v_{z}, u_{z}-w_{x}, v_{x}-u_{y}\right) .
$$

The evolution of the vorticity is governed by the equation

$$
\Omega_{t}+(\mathbf{u} \cdot \nabla) \Omega-2 \omega\left(u_{y}, v_{y}, w_{y}\right)=(\Omega \cdot \nabla) \mathbf{u} .
$$

Remark 2.1 Throughout the paper we shall work under the assumption that $\Omega_{2}+2 \omega \neq 0$. This is a reasonable assumption, since $\omega \approx 0.73 \times 10^{-4} \mathrm{rad} / \mathrm{s}$, while a typical value for $\Omega_{2}$ in the equatorial Pacific is $25 \times 10^{-3} s^{-1}$, cf. [4].

After these preliminary considerations we are able to formulate the main result of the paper.

Theorem 2.2 Gravity wave trains can propagate at the free surface of a water flow over a flat bed governed by Eqs. (2.1)-(2.5) and exhibiting a constant vorticity only if the flow is two-dimensional.

Proof We start the proof by noticing that, due to the assumption of constant vorticity, the evolution equation (2.7) takes the form

$$
(\Omega \cdot \nabla) \mathbf{u}+2 \omega\left(u_{y}, v_{y}, w_{y}\right)=0 .
$$

We are going to use the third equation in the system above, which can be written as

$$
\Omega_{1} w_{x}+\left(\Omega_{2}+2 \omega\right) w_{y}+\Omega_{3} w_{z}=0,
$$


to prove that $\Omega_{3}=0$. We will resort to the method of proof by contradiction and assume that $\Omega_{3} \neq 0$. Consequently, $w$ is constant in the direction of the vector $\left(\Omega_{1}, \Omega_{2}+2 \omega, \Omega_{3}\right)$, which, owing to $\Omega_{3} \neq 0$, is not parallel to the flat bed $z=-d$. We then use the kinematic condition (2.4) and argue as in [3] to conclude that $w=0$ within the fluid domain. Moreover, $w=0$ implies that $u_{z}=\Omega_{2}$ and $v_{z}=-\Omega_{1}$. The latter two relations yield the existence of two functions $\tilde{u}=\tilde{u}(x, y, t)$ and $\tilde{v}=\tilde{v}(x, y, t)$ such that

$$
\begin{aligned}
u(x, y, z, t) & =\tilde{u}(x, y, t)+\Omega_{2} z, \\
v(x, y, z, t) & =\tilde{v}(x, y, t)-\Omega_{1} z .
\end{aligned}
$$

The advantage of working with $\tilde{u}, \tilde{v}$ emerges as the possibility of deriving from (2.2) the existence of a function $\psi(x, y, t)$ satisfying

$$
\tilde{u}=\psi_{y}, \quad \tilde{v}=-\psi_{x} .
$$

The latter equation can be used to rewrite $\Omega_{3}=v_{x}-u_{y}$ as

$$
\psi_{x x}+\psi_{y y}=-\Omega_{3} .
$$

Moreover, the first two equations of (2.9) can be written in terms of the function $\psi$ as

$$
\begin{aligned}
& \Omega_{1} \psi_{x y}+\left(\Omega_{2}+2 \omega\right) \psi_{y y}+\Omega_{2} \Omega_{3}=0 \\
& -\Omega_{1} \psi_{x x}-\left(\Omega_{2}+2 \omega\right) \psi_{x y}-\Omega_{1} \Omega_{3}=0 .
\end{aligned}
$$

Note that the system (2.13) implies that $\Omega_{1}^{2}+\left(\Omega_{2}+2 \omega\right)^{2}>0$. Therefore, we obtain

$$
\begin{aligned}
& \psi_{x x}=\frac{-\Omega_{1}^{2} \Omega_{3}-2 \omega\left(\Omega_{2}+2 \omega\right) \Omega_{3}}{\Omega_{1}^{2}+\left(\Omega_{2}+2 \omega\right)^{2}}, \\
& \psi_{x y}=-\frac{\Omega_{1} \Omega_{2} \Omega_{3}}{\Omega_{1}^{2}+\left(\Omega_{2}+2 \omega\right)^{2}}, \\
& \psi_{y y}=-\frac{\Omega_{2}\left(\Omega_{2}+2 \omega\right) \Omega_{3}}{\Omega_{1}^{2}+\left(\Omega_{2}+2 \omega\right)^{2}},
\end{aligned}
$$

relations from which we infer that

$$
\psi(x, y, t)=D x^{2}+E x y+F y^{2}+d(t) x+e(t) y+f(t),
$$

where

$$
\begin{aligned}
& D=\frac{1}{2} \cdot \frac{-\Omega_{1}^{2} \Omega_{3}-2 \omega\left(\Omega_{2}+2 \omega\right) \Omega_{3}}{\Omega_{1}^{2}+\left(\Omega_{2}+2 \omega\right)^{2}}, \\
& E=-\frac{\Omega_{1} \Omega_{2} \Omega_{3}}{\Omega_{1}^{2}+\left(\Omega_{2}+2 \omega\right)^{2}}, \\
& F=-\frac{1}{2} \cdot \frac{\Omega_{2}\left(\Omega_{2}+2 \omega\right) \Omega_{3}}{\Omega_{1}^{2}+\left(\Omega_{2}+2 \omega\right)^{2}},
\end{aligned}
$$

and $d, e$ and $f$ are functions. With the previous considerations in mind, the kinematic boundary condition (2.3) becomes

$$
\left[-c+E x+2 F y+e(t)+\Omega_{2} \eta(x-c t)\right] \eta^{\prime}(x-c t)=0 .
$$

Since $\eta^{\prime}$ is not identically zero and the equality in (2.16) is true for all $x$ it follows from above that $F=0$. Since $\Omega_{2}+2 \omega \neq 0$, it necessarily follows from $F=0$ that $\Omega_{2}=0$, because we 
are also in the hypothesis that $\Omega_{3} \neq 0$. Moreover, we also have that $E=0$ and $D=-\frac{1}{2} \Omega_{3}$. Consequently, from relation (2.16) we conclude that for all $x$ it holds

$$
(e(t)-c) \eta^{\prime}(x-c t)=0,
$$

which implies that $e(t) \equiv c$, and therefore

$$
\begin{aligned}
& u(x, y, z, t) \equiv c, \\
& v(x, y, z, t)=-d(t)+\Omega_{3} x-\Omega_{1} z, \\
& w(x, y, z, t)=0 .
\end{aligned}
$$

From the third of Euler's equations (2.1) we conclude that

$$
P_{z}=\rho(2 \omega c-g) \text { throughout the fluid, }
$$

which implies that

$$
P(x, y, z, t)=\rho(2 \omega c-g) z+p(x, y, t),
$$

for some function $p(x, y, t)$. From (2.17) and the first of the Euler's equations we obtain that $p_{x}=P_{x}=0$, while using also the second of the Euler's equations we get that $p_{y}=P_{y}=$ $\rho\left(d^{\prime}(t)-c \Omega_{3}\right)$. Consequently,

$$
P(x, y, z, t)=\rho\left(d^{\prime}(t)-c \Omega_{3}\right) y+\rho(2 \omega c-g) z+\tilde{f}(t),
$$

for some function $\tilde{f}$. The dynamic boundary condition (2.5) can now be written

$$
\rho\left(d^{\prime}(t)-c \Omega_{3}\right) y+\tilde{f}(t)=P_{a t m}+\rho(g-2 \omega c) \eta(x-c t), \text { for all } x, y, t .
$$

We infer from above that the coefficient of $y$ has to vanish and that $\tilde{f}$ and $\eta$ have to be constant functions. But $\eta$ constant is equivalent with our flow having a flat surface, which is a contradiction.

The contradiction that we reached shows that $\Omega_{3}=0$. In the following, we will be concerned with proving that $\Omega_{1}$ also vanishes. Moreover, we will show that the pressure $P$ and the velocity field $(u, v, w)$ are independent of $y$.

Our next goal is to show that $\Omega_{1}=0$ and for this purpose we assume for the sake of contradiction that $\Omega_{1} \neq 0$.

We note first that from (2.8) and using that $\Omega_{3}=0$ we obtain

$$
\begin{aligned}
& \Omega_{1} u_{x}+\left(\Omega_{2}+2 \omega\right) u_{y}=0, \\
& \Omega_{1} v_{x}+\left(\Omega_{2}+2 \omega\right) v_{y}=0, \\
& \Omega_{1} w_{x}+\left(\Omega_{2}+2 \omega\right) w_{y}=0 .
\end{aligned}
$$

On the other hand, from $0=\Omega_{3}=v_{x}-u_{y}$ we obtain a function $\varphi=\varphi(x, y, z, t)$ which satisfies

$$
u=\varphi_{x}, \quad v=\varphi_{y} .
$$

Up to addition to $\varphi$ of a function that depends only on $z$ and $t$ we infer from the expressions of $\Omega_{1}$ and $\Omega_{2}$ that

$$
w=\varphi_{z}-\left(\Omega_{2} x-\Omega_{1} y\right) .
$$

With the help of the above expressions of $u, v, w$ in terms of $\varphi$ we see that the equation of mass conservation (2.2) becomes

$$
\varphi_{x x}+\varphi_{y y}+\varphi_{z z}=0 .
$$


The harmonicity of $w$ emerges now as a direct consequence of differentiating (2.22) with respect to $z$. We are going to show now that $w=0$ on a plane parallel to the flat bed and contained entirely in the fluid domain. To be more precise, let

$$
l:=\left\{\left(x_{0}, y, \eta\left(x_{0}, t_{0}\right)\right): y \in \mathbb{R}\right\}
$$

be a trough line located at some position $x_{0}$ at some moment $t_{0}$. Using the kinematic condition (2.3) and the fact that $\eta_{x}=0$ along $l$ we conclude that

$$
w=0 \text { on the whole of } l .
$$

The third relation in (2.21) allows us to conclude that $w$ is constant in the direction of the vector $\left(\Omega_{1}, \Omega_{2}+2 \omega, 0\right)$ which, due to the assumption $\Omega_{1} \neq 0$, is not collinear with $l$. Thus, $w=0$ at all points of the plane containing $l$ and spanned by $l$ and the vector $\left(\Omega_{1}, \Omega_{2}+2 \omega, 0\right)$, which is in fact the plane $z=\eta\left(x_{0}, t\right)$ and which is parallel to the bed $z=-d$ and contained entirely in the fluid domain.

Using that $w$ is a bounded harmonic function that vanishes on $z=-d$ and on $z=\eta\left(x_{0}, t\right)$ we conclude from the Phragmen-Lindelöf maximum principle, cf. [15], that $w=0$ in the region of the fluid domain bounded by the flat bed and the plane $z=\eta\left(x_{0}, t\right)$. We can also infer from [14] that, owing to its harmonicity, $w$ is real-analytic at any instant $t$. Thus, since $w=0$ on an open set, it must vanish on the entire fluid domain. Then, as in the beginning of the proof we infer the existence of a function $\psi(x, y, t)$ satisfying relations (2.10) and (2.11). In addition, since now $\Omega_{3}=0$, we have for the function $\psi$ the relations

$$
\psi_{x x}+\psi_{y y}=0
$$

and

$$
\begin{aligned}
& \Omega_{1} \psi_{x y}+\left(\Omega_{2}+2 \omega\right) \psi_{y y}=0 \\
& -\Omega_{1} \psi_{x x}-\left(\Omega_{2}+2 \omega\right) \psi_{x y}=0 .
\end{aligned}
$$

We obtain immediately from (2.24) and (2.25) that

$$
\psi_{x x}=\psi_{x y}=\psi_{y y}=0
$$

within the fluid domain which means that there are function $d(t), e(t)$ and $f(t)$ such that

$$
\psi(x, y, t)=d(t) x+e(t) y+f(t)
$$

for all $x, y, t$. We rely once more on the kinematic condition (2.3) to derive the relation

$$
\left[-c+e(t)+\Omega_{2} \eta(x-c t)\right] \eta^{\prime}(x-c t)=0 .
$$

From the latter we infer first that $\Omega_{2}=0$ and then, as a consequence, it follows that $e(t) \equiv c$. We follow now the same route as in the beginning of the proof and reach that $\eta$ has to be a constant. The latter contradiction shows that $\Omega_{1}$ necessarily has to vanish.

We are going to prove now that the velocity field does not depend on the $y$ variable. Moreover, we will prove that $v$ is in fact a constant and the $y$-derivative of the pressure function vanishes. To this end note that the vorticity equation (2.8) takes the form

$$
\left(\Omega_{2}+2 \omega\right) u_{y}=\left(\Omega_{2}+2 \omega\right) v_{y}=\left(\Omega_{2}+2 \omega\right) w_{y}=0,
$$

which, due to $\Omega_{2}+2 \omega \neq 0$, implies $u_{y}=v_{y}=w_{y}=0$. Using also $0=\Omega_{1}=w_{y}-v_{z}$ and $0=\Omega_{3}=v_{x}-u_{y}$ we infer that $v_{x}=v_{z}=0$. Thus, the second component of the velocity field depends only on $t$. 
A consequence of the previous findings is that the second equation in (2.1) reduces to

$$
v_{t}=-\frac{1}{\rho} P_{y} .
$$

Integrating with respect to $y$ in the above relation we find that

$$
P(x, y, z, t)=-\rho v_{t} y+F(x, z)+R(t),
$$

for some functions $F$ and $R$ whose variables are as indicated in the formula above. With the latter structure for $P$, the dynamic boundary condition (2.5) becomes

$$
-\rho v_{t} y+F(x, \eta(x))+R(t)=P_{\mathrm{atm}},
$$

for all $x, y$ and $t$. But this implies that $v_{t} \equiv 0$. Therefore, $P_{y} \equiv 0$. We have thus proved that the flow has a two-dimensional character.

Remark 2.3 Note that, due to the assumption $\Omega_{2}+2 \omega \neq 0$ - reasonable in the setting of equatorial waves - we can dispense of the constant nonzero vorticity requirement made in [2] in the context of capillary wave trains, in [3] for the case of gravity wave trains and in [26] regarding capillary-gravity water waves.

Acknowledgements Open access funding provided by University of Vienna. We are thankful to the anonymous referee for many useful suggestions that considerably improved the exposition.

Open Access This article is distributed under the terms of the Creative Commons Attribution 4.0 International License (http://creativecommons.org/licenses/by/4.0/), which permits unrestricted use, distribution, and reproduction in any medium, provided you give appropriate credit to the original author(s) and the source, provide a link to the Creative Commons license, and indicate if changes were made.

\section{References}

1. Constantin, A.: On the deep water wave motion. J. Phys. A 34, 1405-1417 (2001)

2. Constantin, A., Kartashova, E.: Effect of non-zero constant vorticity on the nonlinear resonances of capillary water waves. Europhys. Lett. 86, 29001 (2009)

3. Constantin, A.: Two-dimensionality of gravity water flows of constant non-zero vorticity beneath a surface wave train. Eur. J. Mech. B Fluids 30, 12-16 (2011)

4. Constantin, A.: On the modelling of equatorial waves. Geophys. Res. Lett. 39, L05602 (2012)

5. Constantin, A.: An exact solution for equatorially trapped waves. J. Geophys. Res. Oceans 117, C05029 (2012)

6. Constantin, A., Germain, P.: Instability of some equatorially trapped waves. J. Geophys. Res. Oceans $\mathbf{1 1 8}$, 2802-2810 (2013)

7. Constantin, A.: Some three-dimensional nonlinear equatorial flows. J. Phys. Oceanogr. 43(1), 165-175 (2013)

8. Constantin, A.: Some nonlinear, equatorially trapped, nonhydrostatic internal geophysical waves. J. Phys. Oceanogr. 44(2), 781-789 (2014)

9. Constantin, A., Johnson, R.S.: The dynamics of waves interacting with the equatorial undercurrent. Geophys. Astrophys. Fluid Dyn. 109(4), 311-358 (2015)

10. Constantin, A., Johnson, R.S.: An exact, steady, purely azimuthal equatorial flow with a free surface. J. Phys. Oceanogr. 46(6), 1935-1945 (2016)

11. Constantin, A., Johnson, R.S.: An exact, steady, purely azimuthal flow as a model for the Antarctic circumpolar current. J. Phys. Oceanogr. 46(12), 3585-3594 (2016). doi:10.1175/JPO-D-16-0121.1

12. Cushman-Roisin, B., Beckers, J.-M.: Introduction to Geophyiscal Fluid Dynamics: Physical and Numerical Aspects. Academic Press, Waltham (2011)

13. Dubreil-Jacotin, M.-L.: Sur les ondes de type permanent dans les liquides hétérogène. Atti Accad. Naz. Lincei, Mem. Cl. Sci. Fis. Mat. Nat. 15, 814-819 (1932)

14. Evans, L.C.: Partial Differential Equations. American Mathematical Society, Providence (1998) 
15. Fraenkel, L.E.: An Introduction to Maximum Principles and Symmetry in Elliptic Problems. Cambridge University Press, Cambridge (2000)

16. Genoud, F., Henry, D.: Instability of equatorial water waves with an underlying current. J. Math. Fluid Mech. 16(4), 661-667 (2014)

17. Gerstner, F.: Theorie der Wellen samt einer daraus abgeleiteten Theorie der Deichprofile. Ann. Phys. 2, 412-445 (1809)

18. Henry, D.: On gerstner's water wave. J. Nonlinear Math. Phys. 15(suppl. 2), 87-95 (2008)

19. Henry, D.: An exact solution for equatorial geophysical water waves with an underlying current. Eur. J. Mech. B Fluids 38, 18-21 (2013)

20. Henry, D., Hsu, H.-C.: Instability of internal equatorial water waves. J. Differ. Equ. 258(4), 1015-1024 (2015)

21. Henry, D.: Exact equatorial water waves in the $f$-plane. Nonlinear Anal. Real World Appl. 28, 284-289 (2016)

22. Henry, D.: Equatorially trapped nonlinear water waves in a $\beta$-plane approximation with centripetal forces. J. Fluid Mech. 804, R1 (2016). doi:10.1017/jfm.2016.544

23. Ionescu-Kruse, D.: Instability of equatorially trapped waves in stratified water. Ann. Mat. Pura Appl. (4) 195(2), 585-599 (2016)

24. Ionescu-Kruse, D., Martin, C. I.: Local stability for an exact steady purely azimuthal equatorial flow, to appear in J. Math. Fluid Mech. doi:10.1007/s00021-016-0311-4

25. Hsu, H.-C., Martin, C.I.: Free-surface capillary-gravity azimuthal equatorial flows. Nonlinear Anal. 144, 1-9 (2016)

26. Martin, C.I.: Resonant interactions of capillary-gravity water waves. J. Math. Fluid Mech. (2016). doi:10. 1007/s00021-016-0306-1

27. Matioc, A.-V.: An exact solution for geophysical equatorial edge waves over a sloping beach. J. Phys. A 45(36), 365501, 10 (2012)

28. Matioc, A.-V.: Exact geophysical waves in stratified fluids. Appl. Anal. 92(11), 2254-2261 (2013)

29. Stuhlmeier, R.: On constant vorticity flows beneath two-dimensional surface solitary waves. J. Nonlinear Math. Phys 19(suppl. 1), 1240004 (2012)

30. Wahlén, E.: Non-existence of three-dimensional travelling water waves with constant non-zero vorticity. J. Fluid Mech. 746, R2 (2014) 